\title{
NECESSARY CONDITIONS FOR DIVERGENCE IN LINEAR ELASTIC SYSTEMS*
}

\author{
By J. A. WALKER (Northwestern University)
}

Consider a linear elastic system without velocity-dependent forces,

$$
M \ddot{y}(t)+K y(t)=0
$$

such that for all $\tau \in[0, \infty),(y(\tau), \dot{y}(\tau)) \in \mathcal{Y} \times \mathcal{Y}$, where

(i) (.) denotes $d() / d t$ and the time $t \in[0, \infty)$,

(ii) $\mathfrak{H}$ is a real Hilbert space with inner product $\langle$,$\rangle , and \mathcal{Y}$ is dense in $\mathfrak{H}$,

(iii) the real linear spatial operators $M$ and $K$ are independent of time, $K^{-1}$ exists, and $\mathfrak{D}_{K}=\mathcal{Y}, \mathfrak{R}_{K}=\mathfrak{H C}, D_{M}=\mathbb{R}_{M}=\mathfrak{H C}$.

Thus the elastic system may be lumped-parameter, distributed-parameter, or a combination of the two.

The stability of the equilibrium of a system similar to this, but including velocitydependent forces, was studied by the direct method of Liapunov in [1]. Often it is also of interest to distinguish between two modes of instability in system (1), these modes being known as "divergence" and "flutter" $[2,3,4]$. A divergent solution is of the form

$$
y(t)=y_{0} \exp (\rho t), \quad y_{0} \in \mathcal{Y},
$$

where $\rho$ is a positive real, while a flutter solution is of the form

$$
y(t)=\exp (\rho t)\left(y_{1} \sin \omega t+y_{2} \cos \omega t\right), \quad y_{1}, y_{2} \in \mathcal{Y},
$$

or

$$
y(t)=t\left(y_{1} \sin \omega t+y_{2} \cos \omega t\right), \quad y_{1}, y_{2} \in \mathcal{Y},
$$

where $\rho$ and $\omega$ are positive real.

If $M$ and $K$ are symmetric and $M$ is positive definite, system (1) is termed conservative and the functionals $T(\dot{y}), U(y)$, defined by

$$
\begin{array}{ll}
2 T(\dot{y})=\langle\dot{y}, M \dot{y}\rangle, & \dot{y} \in \mathcal{Y}, \\
2 U(y)=\langle y, K y\rangle, & y \in \mathcal{Y},
\end{array}
$$

are known as the kinetic and potential energies, respectively. It is known that flutter does not occur at all in a conservative system, and divergence can occur if, and only if, at least one of the above functionals is not non-negative. It is easily shown that this condition remains necessary for divergence even when $M$ and $K$ are not symmetric.

Theorem: If both $T(\dot{y})$ and $U(y)$ are positive definite, divergence does not occur. ${ }^{1}$

* Received November 2, 1972.

1 A similar statement was recently made for a special case of (1), although the proof was not rigorous [4]. 
Proof: Assume that divergence does occur and both $T(\dot{y})$ and $U(y)$ are positive definite. Then, using (2) and (1), we find

$$
\left(\rho^{2} M+K\right) y_{0}=0,
$$

and thus

$$
\rho^{2}\left\langle y_{0}, M y_{0}\right\rangle=-\left\langle y_{0}, K y_{0}\right\rangle
$$

which contradicts the assumption that both $T(\dot{y})$ and $U(y)$ are positive definite.

The following statement is similarly obvious:

Corollary: If there exists an operator $L, y \subset D_{L}, \mathbb{R}_{L} \subset \mathfrak{F}$, such that both the functionals $\langle L \dot{y}, M \dot{y}\rangle$ and $\langle L y, K y\rangle$ are positive definite, divergence does not occur.

This very simple theorem and its corollary often provide a much easier means of checking for divergence than do the usual eigenvalue or Galerkin approximation methods when studying nonconservative systems. For example, consider the following generalization of the Pflüger problem $[5,6]$ :

$$
\begin{aligned}
& \ddot{y}+\left(\partial^{4}+f(x) \partial^{2}\right) y=0, \quad 0<x<1, \\
& \mathcal{Y}=\left\{y \in \mathcal{K}_{2}^{4}(0,1) \mid y=\partial^{2} y=0 \text { at } x=0,1\right\}, \\
& \mathcal{H}=\mathscr{L}_{2}(0,1) .
\end{aligned}
$$

Using the corollary and choosing $L=-\partial^{2}$, we find

$$
\begin{aligned}
& \langle L \dot{y}, M \dot{y}\rangle=-\int_{0}^{1} \dot{y} \partial^{2} \dot{y} d x=\int_{0}^{1}(\partial \dot{y})^{2} d x \\
& \langle L y, K y\rangle=-\int_{0}^{1}\left(\partial^{2} y\right)\left(\partial^{4}+f(x) \partial^{2}\right) y d x=\int_{0}^{1}\left[\left(\partial^{3} y\right)^{2}-f(x)\left(\partial^{2} y\right)^{2}\right] d x,
\end{aligned}
$$

and divergence does not occur provided the operator

$$
\begin{aligned}
B & =-\left(\partial^{2}+f(x)\right), \\
\mathscr{D}_{B} & =\left\{u \in \mathcal{F}_{2}^{2}(0,1) \mid u=0 \text { at } x=0,1\right\}
\end{aligned}
$$

is positive definite. In [6] it was shown that this condition was actually sufficient for stability of the equilibrium of (6) when an appropriate stability norm is used.

The theorem has a particularly simple physical interpretation when $K$ and $M$ are such that $y \subset D_{K^{*}}, y \subset D_{M^{*}}$, where $K^{*}$ and $M^{*}$ are the adjoints of $K$ and $M$, respectively. In this case

$$
\begin{aligned}
& 4 T(\dot{y})=\left\langle\dot{y},\left(M+M^{*}\right) \dot{y}\right\rangle, \quad \dot{y} \in \mathcal{Y}, \\
& 4 U(y)=\left\langle y,\left(K+K^{*}\right) y\right\rangle, \quad y \in \mathcal{Y},
\end{aligned}
$$

and thus divergence may occur in (1) only if divergence occurs in the system

$$
\left(M+M^{*}\right) \ddot{y}+\left(K+K^{*}\right) y=0, \quad(y, \dot{y}) \in \mathcal{Y} \times \mathcal{Y} .
$$

Note that (10) is conservative if $M$ is positive definite.

There is a similar physical interpretation of the corollary if $L$ is chosen such that $\mathfrak{D}_{L}{ }^{*}=\mathfrak{H C}, L(\mathcal{Y}) \subset \mathfrak{D}_{K^{*}}$. In this case 


$$
\begin{gathered}
2\langle L \dot{y}, M \dot{y}\rangle=\left\langle\dot{y}\left(L^{*} M+M^{*} L\right) \dot{y}\right\rangle, \quad \dot{y} \in \mathcal{Y}, \\
\left.2\langle L Y, K y\rangle=\left\langle y, L^{*} K+K^{*} L\right) y\right\rangle, \quad y \in \mathcal{Y},
\end{gathered}
$$

and thus divergence may occur in (1) only if divergence occurs in the system

$$
\left(L^{*} M+M^{*} L\right) \ddot{y}+\left(L^{*} K+K^{*} L\right) y=0, \quad(y, \dot{y}) \in \mathcal{Y} \times \mathcal{Y} .
$$

Note that (12) is conservative if $L^{*} M$ is positive definite.

To demonstrate this analogy consider the previous example and choose $L=B^{-1}$. Then

$$
L^{*} M+M^{*} L=2 B^{-1}, \quad L^{*} K+K^{*} L=-2 \partial^{2},
$$

and (12) becomes

$$
B^{-1} \ddot{y}-\partial^{2} y=0, \quad(y, \dot{y}) \in \mathcal{Y} \times \mathcal{Y},
$$

which is conservative provided $B^{-1}$ is positive definite.

Now let us consider the effect of velocity-dependent forces by replacing (1.1) with

$$
M \ddot{y}(t)+C \dot{y}(t)+K y(t)=0, \quad(y, \dot{y}) \in \mathcal{Y} \times \mathcal{Y},
$$

where $M, K, \mathcal{Y}$, and $\mathcal{H}$ are as previously defined while $C$ is a real linear spatial operator, independent of time, such that $D_{C}=\mathcal{Y}, \mathfrak{R}_{C} \subset \mathfrak{F}$. Again considering the possibility of a divergent solution (2), we find the following:

Theorem: If both $T(\dot{y})$ and $U(y)$ are positive definite, while $\langle\dot{y}, C \dot{y}\rangle$ is non-negative, divergence does not occur.

Proof: Assume that divergence does occur, both $T(\dot{y})$ and $U(y)$ are positive definite, and $\langle\dot{y}, C \dot{y}\rangle$ is non-negative. Then using (2) in (15), $\left(\rho^{2} M+\rho C+K\right) y_{0}=0$, and thus $\rho^{2}\left\langle y_{0}, M y_{0}\right\rangle=-\rho\left\langle y_{0}, C y_{0}\right\rangle-\left\langle y_{0}, K y_{0}\right\rangle$, which is a contradiction since $\rho>0$.

Corollary: If there exists an operator $L, \mathcal{Y} \subset D_{L}, \mathfrak{R}_{L} \subset \mathcal{H}$, such that both $\langle L \dot{y}, M \dot{y}\rangle$ and $\langle L y, K y\rangle$ are positive definite while $\langle L \dot{y}, C \dot{y}\rangle$ is nonnegative, divergence does not occur.

This theorem and its corollary generalize the preceding results to nonconservative systems with velocity-dependent forces and provide, in effect, simple sufficient conditions for the absence of divergence. At present there are no equally simple sufficient conditions for the absence of flutter. It should also be noted that although the presence of divergence or flutter implies instability of the equilibrium relative to any given stability norm

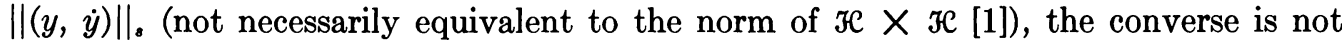
generally true unless $\mathfrak{H C}$ is finite-dimensional [7].

\section{REFERENCES}

[1] J. A. Walker, Energy-like Liapunov functionals for linear elastic systems on a Hilbert space, Quart. Appl. Math. 30, 465-470 (1973)

[2] H. Ziegler, Die Stabilitätskriterion der Elastomechanik, Ingenieur-Archiv 20, 49-56 (1952)

[3] H. Ziegler, Principles of structural stability, Blaisdell, 1968

[4] H. H. E. Leipholz, On the sufficiency of the energy criterion for the stability of certain nonconservative systems of the follower-load type, ASME J. Appl. Mech. 32, 717-722 (1972)

[5] A. Pflüger, Stabilitätsprobleme der Elastostatik, Springer-Verlag, 1964

[6] J. A. Walker, Liapunov analysis of the generalized Pfiüger problem, ASME J. Appl. Mech. 39, 935-938 (1972)

[7] A. M. Mochvan, The direct method of Liapunov in stability of elastic systems, Prik. Mat. Mech. 23 (1959), 483-493 